\title{
Remote Sensing in Decision Support Systems: Using Fuzzy Post Adjustment in Localisation of Weed Prediction
}

\author{
\#Andrew Chiou ${ }^{1}$ and Xinghuo $\mathrm{Yu}^{2}$ \\ ${ }^{1}$ School of Computing Sciences, Central Queensland University \\ Rockhampton,4702 Qld, Australia, a.chiou@cqu.edu.au \\ ${ }^{2}$ School of Electrical and Computer Engineering, RMIT University \\ Melbourne,3001Victoria, Australia, x.yu@rmit.edu.au
}

\begin{abstract}
This paper explores the post adjustment of input data from a remote source to fit localised weed prediction for the control and management of weed infestation. The deployment of decision support systems in agricultural sectors often require refinement of its results to adapt to data that has been acquired externally via remote sensing. This paper will detail the fuzzy meta-consequent functions to facilitate the post adjustment. A case study is presented to demonstrate the workability of such fuzzy post-adjustment in the prediction of weed infestation.
\end{abstract}

\section{INTRODUCTION}

Parthenium weed (Parthenium hysterophorus L.) infestation has demonstrated the ability to cause significant environmental, health and financial problems if not managed properly in areas occupied by agricultural industries. Its rapid spread has covered $170,000 \mathrm{~km} 2(10 \%)$ of Queensland, Australia [1]. Economically, the uncontrolled spread of parthenium weed ${ }^{1}$ has the potential to cause losses of $A \$ 109$ 129 million annually [2]. One of the primary steps taken to curb this infestation is by providing expert advice on the best control and management strategies to stakeholders concerned. One of the strategies requires the prevention of new infestation and the eradication of known infestation. To accomplish this, an accurate prediction of the weed spread is needed to allow stakeholders to take the necessary preventive measures to curb further dispersal. An agriculture decision support system, P-Expert, has been developed to provide advice on control and management strategies [3]. This paper will investigate the implementation of a function employing fuzzy meta-consequent function to allow data from in-coming external source to be adapted to localised conditions. This is followed by a case study demonstrating the feasibility of providing a reliable weed prediction based on local data.

1 'Parthenium weed' is always referred to by its complete name in both literature and actual practice.

\section{BACKGROUND}

Figure 1 shows the general framework of P-Expert, the decision support system. Details of it implementation and deployment has been previously described in [3-5]. The following subsections will elaborate on P-Expert's process flow in detail based on the knowledge acquisition and consultation process levels.

\section{A. Knowledge Acquisition Level}

The knowledge acquisition level involves the procedure and relevant databases as indicated by the shaded area on the right. The clientele at this phase typically involve both knowledge engineers and end users, which will participate in the preparation of the knowledge-rule, external and discourse databases in P-Expert. In preparation of the knowledge-rule database, the knowledge engineers will firstly elicit the necessary knowledge and information in regards to best management practices on parthenium weed. This knowledge is subsequently transformed into fuzzy membership models that are appended, using the rule editors at the editor layer, into the appropriate knowledge and If-Then rule databases. In addition, knowledge engineers will also be involved in the preparation and maintenance of the discourse base by formulating and drawing relationships between rules and the required explanation. The preparation of the external base is unique. In contrast with the knowledge-rule base which is typically consistent throughout the lifespan of the main system, the incoming data to the external base is dynamic and varies with time and type of local trends. This database is only instantiated (i.e. going 'live') at the instant the consultation process takes place (as shown in shaded area on the left-hand side in Figure 1). The validity of the data in the external base has a predefined lifetime that can expire depending on the requirements of the end user. Note that the process flow of the intermediate preparation of the external base takes place at the consultation process. There are external data that does not have a limited lifespan, but nonetheless may still require periodical updates. These are indicated in Figure 1 by the external readings of incoming data sourced from satellite imagery, weather reports and remote sensors [6]. 


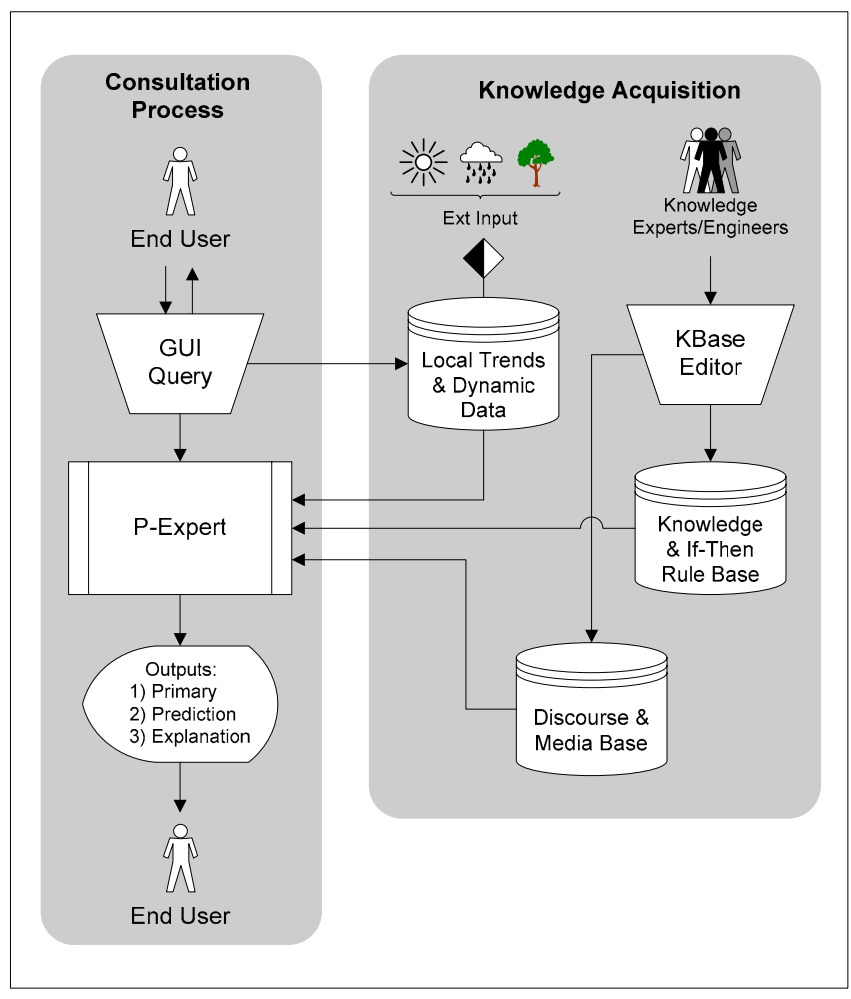

Fig.1: P-Expert's process flow showing the interaction and relationship between groups of clientele/entities on two different operational levels.

\section{B. Consultation Process Level}

The process flow in the consultation process involving the relevant procedures is indicated in the shaded area on the lefthand side in Figure 1. This activity involves direct interaction between the end user and P-Expert. Fundamentally, the end user provides answers in response to P-Expert's queries. As will be shown later in the test case in this paper, these queries relates to local trends required for the meta post-adjustment inference process. These dynamic responses (i.e. input data) are processed immediately and recorded for future references. However as explained in the previous subsection, these data have a limited lifespan and is subjected to expiration.

Once end users have supplied all required responses to $\mathrm{P}$ Expert, the data gathered are then inferred by the core engine and a final outcome is provided. At the end of the consultation process, the final output should be composed of the four required primary recommendations and the two supporting outputs. The consultation process between PExpert and end users terminates at this point. Based on the output (i.e. recommendations on strategies for control and management of parthenium weed), the end user will determine what further actions to apply or what advice to put into practice. At this junction, note that there are three different outputs. The primary output has been discussed in detail in [4], the explanatory output in [7], and the prediction of weed dispersal will be the focus of this paper.

\section{Fuzzy Post Adjustment Functions}

The use of fuzzy meta-consequent was initially proposed by the author as a post adjustment mechanism to incorporate external data with data from the main system's knowledgebase to be embedded as an integral part of the system's framework. The post adjustment mechanism allows the system to take into account the data derived from remote sensing, archival records, qualitative descriptions (i.e. anecdotal references, verbal statements) and 'rule of thumb'. The three types of post adjustment described in the following sections are simple, multi and complex consequents.

\section{A. Simple Consequent}

In almost all cases, complex statements from an external source could inevitably cause an exception in conventional fuzzy logic mechanics (e.g. "When temperature is high, weed outbreak is unlikely, unless if it is in wet season, this has the reverse effect locally."). However, using a simple consequent post adjustment, this exception can be modelled a straight forward manner in a one-to-one mapping schema. Appending a $B U T$ operator, the basic fuzzy If-Then rule is now expressed as,

$$
\text { (IF } x \text { is } a \text { THEN } y \text { is } b \text { ) BUT ( } z \text { is } c \text { THEN } y \text { is } d \text { ) }
$$

For example,

(IF temp is high THEN outbreak is unlikely) BUT

(location is wet THEN outbreak is very likely)

Unlike an $E L S E$ operator in regular If-Then rule based expert systems, the BUT operator is always true. That is, the BUT operator is evaluated and executed under all conditions, superseding the consequent of original rule on the LHS of the $B U T$ operator. The simple consequent post adjustment is achieved through domain displacement. The BUT operator affects the shifting of entire domains within the universe of discourse as in Figure 2, in the role of an exception handler. However, this operator comes into effect only if there are applicable local trends disclosed in the data source.

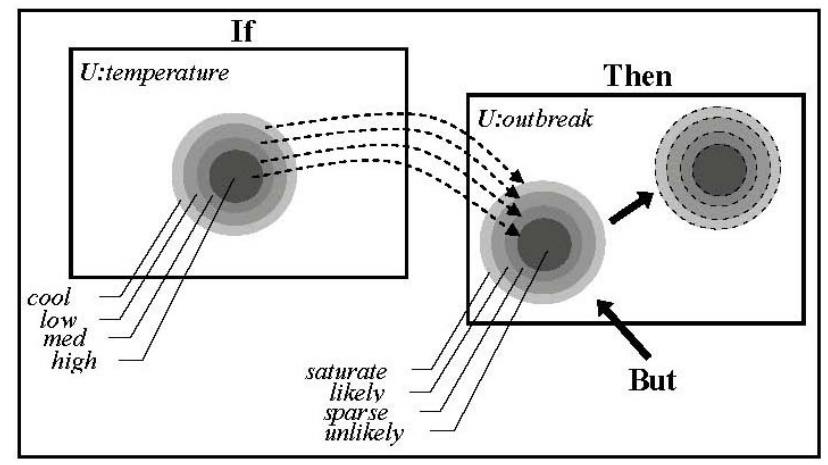

Fig. 2: The BUT operator in a simple consequent If-Then statement handle exceptions in data source by applying domain displacement. 


\section{B. Multi Consequent}

There are however, cases when an exception does not solely occur over membership of domains, but over an entire universe of discourse. This is achieved through a universe displacement (Figure 3). In this instance of one-to-many schema, the multi consequent post adjustment, in the role of redirecting flow control, allows branching to different membership function within the same fuzzy variable. This will allow the same variable to have ownership over different sub-domains. We introduce the operator, $C A S E-O F$, to facilitate the operation of multi consequent post adjustment. The If-Then syntax for the selection is thus,

\section{(IF $x$ is $a$ THEN CASE-OF $m$ )}

\{CASE-OF $m_{1}: y_{l}$ is $b_{l}$;

$$
\text { CASE-OF } \left.m_{n}: y_{n} \text { is } b_{n}\right\}
$$

Where, $m$ is a data source from an external data source (e.g. local trend or exception). An example of (2) can be,

(IF weather is wet THEN CASE-OF location)

\{CASE-OF Mackay: infestation is likely;

CASE-OF Rockhampton: infestation is unlikely;

CASE-OF Gladstone: infestation is unlikely AND flowering is likely\}

Therefore, by applying a $C A S E-O F$ condition on the RHS of the If-Then rule will instantiate and activate different universe of discourse applicable to local trends, as shown in Figure 3.

\section{Complex Consequent}

The complex consequent is a composite of a one-to-one and one-to-many schema. The operators, BUT and CASE-OF are combined to give a mechanism to override the consequent of a rule, and yet facilitating branching under different cases.

The If-Then rule combining (5.2) and (5.3) would give,

(IF $x$ is $a$ THEN $y$ is $b$ ) BUT ( $z$ is $c$ THEN CASE-OF $m$ )

\{CASE-OF $m_{1}: w_{1}$ is $d_{1}$;

$$
\text { CASE-OF } \left.m_{n}: w_{n} \text { is } d_{n}\right\}
$$

Where, $m$ is an external data and the point of redirection. Expanding the previous examples using (3) we have,

(IF temp is high THEN infestation is unlikely) BUT

(location is wet THEN CASE-OF location)

\{CASE-OF Mackay: infestation is likely;

CASE-OF Rockhampton: infestation is unlikely;

CASE-OF Gladstone: infestation is unlikely

AND flowering is likely\}

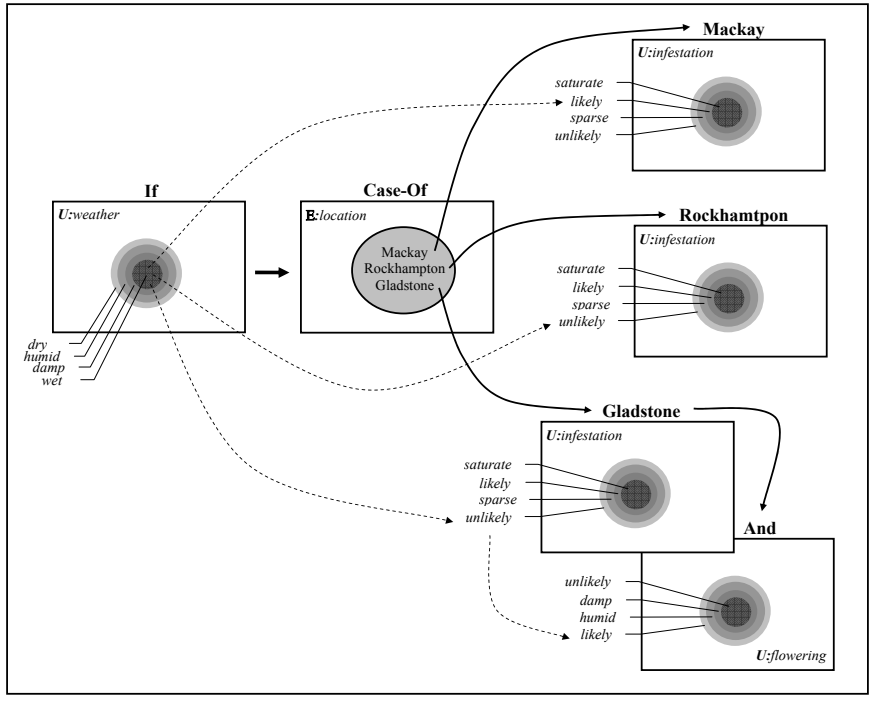

Fig. 3: The $C A S E-O F$ operator in a multi consequent post adjustment will result in universe displacement, initiating branching to different memberships within the same fuzzy variable.

With reference to the above example and to Figure 4, the sequence of events taking place is as follows. In the first phase (indicated by the label 1 in the figure), the basic IfThen rule is initiated. However an exception occurs (at 2), due to a local trend from an external data source that causes the $B U T$ operation to be activated, effectively pre-empting the LHS of the THEN consequent. In a simple consequent post adjustment, a discourse displacement occurs and an alternative consequent is introduced at this stage. In the case of a complex consequent post adjustment (at 3) however, the pre-emption of the LHS of the If-Then rule consequent in (3) is substituted by the $C A S E-O F$ operation, providing a redirection of consequent to a different universe, via the universe displacement meta consequent post adjustment mechanics.

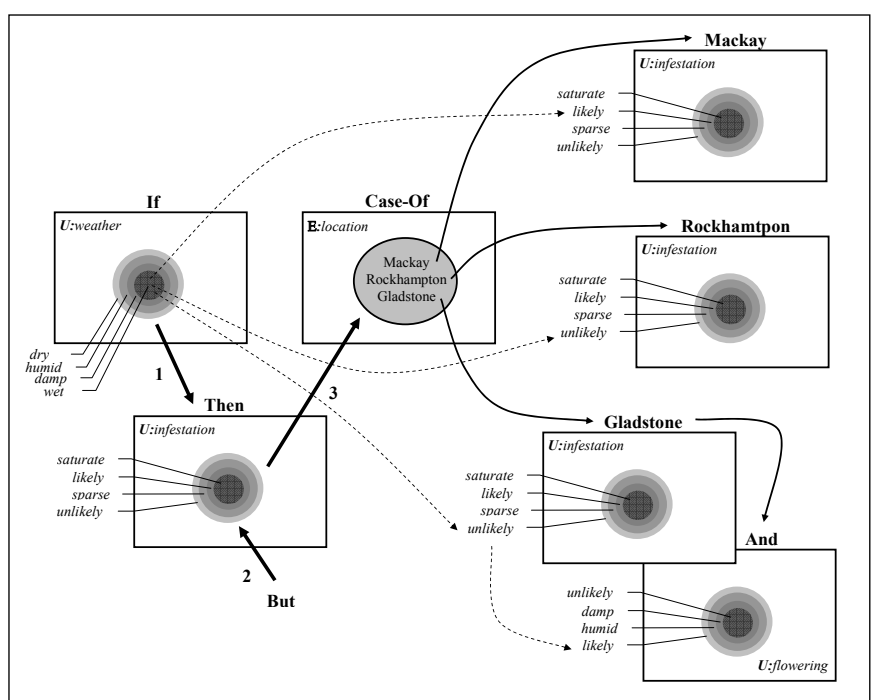

Fig. 4: The complex consequent is a composite of BUT and CASE-OF operators, that causes the pre-emption of the original LHS consequent in the sequence of events labelled 1, 2 and 3. 


\section{IMPLEMENTATION}

The [basic] consequent function, $\mathbf{f}_{\text {conseq }}$, utilising the standard fuzzy inference mechanism can be defined as,

$$
\mathbf{f}_{\text {conseq }}\left(\text { DataSource }_{\text {kbase }}: q_{1}, q_{2} \ldots q_{n}\right)=\left(\text { Output }_{\text {primary }}: a_{1}, a_{2}, a_{3}, a_{4}\right)
$$

where $q_{1}, q_{2} \ldots q_{n}$ are the incoming end user queries submitted to be inferred against the existing data source, DataSource $_{k b a s e}$, available in the main system. This function will return the values $a_{1}, a_{2}, a_{3}$ and $a_{4}$, corresponding with the four primary outputs, as required by the specifications described in [7].

To apply the meta post adjustment mechanism, the function (4) is now extended as,

$$
\begin{aligned}
& \mathbf{f}_{\text {meta_conseq }}\left(\text { DataSource }_{\text {kbase }}: q_{1}, q_{2} \ldots q_{n} \text {, DataSource } \text { ext_source }_{\text {Th }}\right. \text { T) } \\
& =\left(\text { Output }_{\text {primary }}: a_{1}, a_{2}{ }^{\prime}, a_{3}{ }^{\prime}, a_{4}{ }^{\prime}\right)
\end{aligned}
$$

where the two parameters submitted are the original queries as in (4) to be inferred against the existing default data source, DataSource $_{k b a s e}$ and against the external data source, DataSource $_{\text {ext source. }}$ The return values for $\mathbf{f}_{\text {meta_conseq }}$ is now $a_{1}^{\prime}, a_{2}, a_{3}$ ' and $a_{4}$, a localised variant of the original output. And where, $T$ can have following four discrete values: simple_adjustment, multi_adjustment, complex_adjustment and null_adjustment, the first three options respectively refers

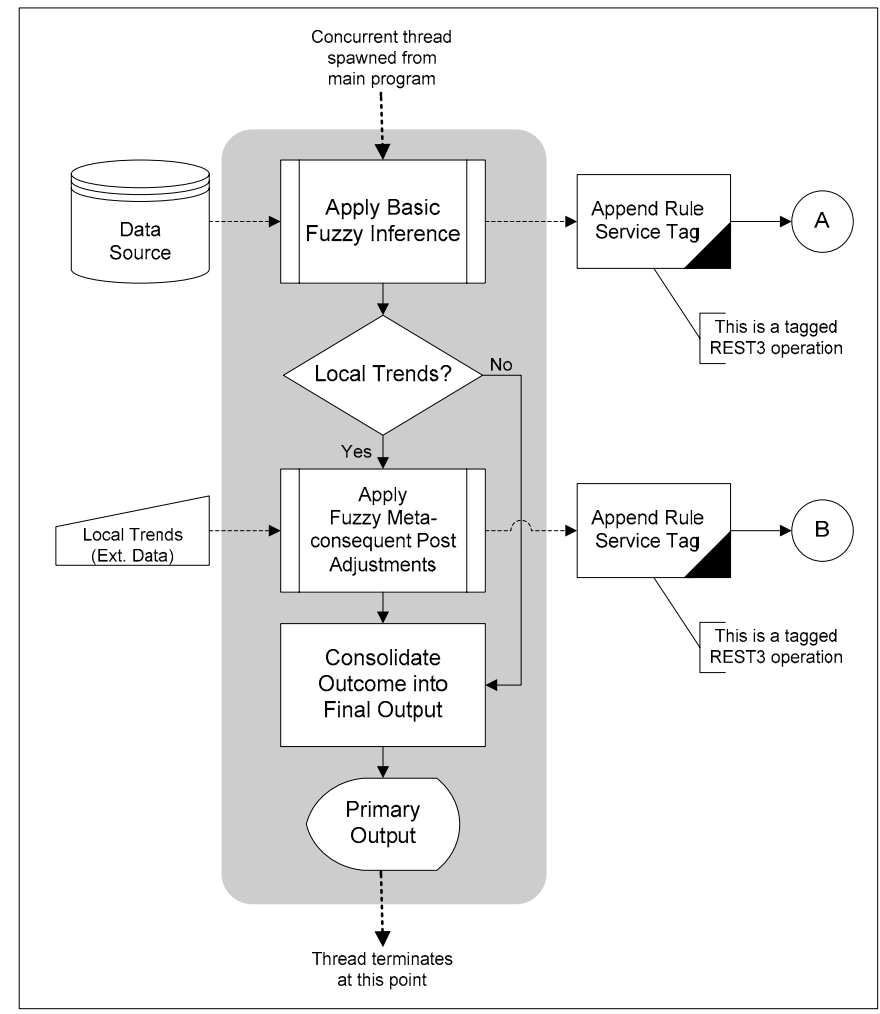

Fig. 5: The program structure detailing the subsystem that provides fuzzy meta-consequent post adjustment functions to the simple consequent, multi consequent and complex consequent post adjustment mechanism to apply. Note the last type, null adjustment, that can be called or perform as a 'catch-all' in cases where a post adjustment is explicitly required, but without the availability of local trend (i.e. external data). In this case the expected output, $a_{1}{ }^{\prime}, a_{2}, a_{3}$ ' and $a_{4}$ ', simply reverts back to the initial intended output, $a_{1}$, $a_{2}, a_{3}$ and $a_{4}$. This provision is to ensure the operational robustness of the main system, that is, it is expected to deliver a reasonable output based on the default data and current knowledgebase in the event that local trends are called for, but are unavailable.

The program implementation of the meta post adjustment functions is shown in Figure 5. This subsection is a concurrent thread spawned by the main program. The dotted lines indicate data and process flow that is outside the control of this subsystem. Note that there are two major functional blocks, apply basic fuzzy inference and apply fuzzy metaconsequent post adjustments, respectively assigned to process functions defined in (4) and (5). The service tags referred to in the figure are to facilitate the discourse semantics (i.e. explanatory capability) REST3 subsystem disclosed in [8].

\section{WeEd PREdiction}

This section demonstrates the methodology on how weed population can be predicted using If-Then rule based fuzzy inference as applied to GIS spatial image. The forecasting mechanism is carried out on spatial images of known infested sites and growth influence factors (e.g. rivers, roads, soil type). These spatial images are separated and categorised into individual layers, where each layer is further sub-classified into themes. By applying a fuzzy membership function onto each of these themes, it is possible to aggregate the individual values into a final weed infestation factor. This allows weed prediction at a large-scale level, as well as at a localised level. By allowing qualitative data (e.g. anecdotal references, local information) to be included in the forecasting process, the fuzzy logic mechanism will be able to utilise it to refine largescale forecasts to obtain localised forecasts. The overall methodology presented in this section covers four phases: pre-processing, thematic forecast, global forecast and local forecast.

\section{A. Phase 1: Pre-processing}

This step involves preparing the proper data structures to facilitate analysis of the spatial image datasets. Adhering to standard GIS practices [9] each layer comprise of themes, $\tau_{1}$, $\tau_{2} \ldots \tau_{\mathrm{n}}$. Themes are made up of features, $\mathrm{Fs}_{1}, \mathrm{Fs}_{2} \ldots F \mathrm{~s}_{\mathrm{n}}$, indicating natural or man-made phenomenon found in maps (e.g. rivers, roads, buildings). In practice, this method of structuring spatial image helps accommodate fuzzy variables and membership functions. The fuzzy representation is: 1) All layers represent a universe of discourse, and 2) Each theme represents a fuzzy variable. 
There are two special themes, $T_{1}$ and $\tau_{\text {null }} . \quad T_{1}$, is a theme reserved for spatial images representing known locations of infested sites. While, $\tau_{\text {null }}$ is a reserved theme for regions labelled as null-zones. Null-zones areas are non-negotiable regions, where no known plant species will propagate at all. The required themes are as follow:

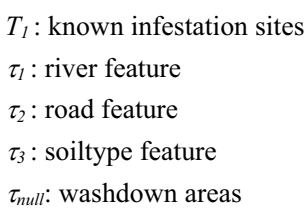

\section{B. $\quad$ Phase 2: Thematic Forecast}

This phase requires that a thematic forecast be carried out individually on each $\tau_{1}, \tau_{2}$ and $\tau_{3}$. In doing so, each factor that influences the propagation of parthenium weed can be segregated from other factors. Forecasting is performed layer by layer on each corresponding cell to determine its infestation factor, that is, a fuzzy consequent membership value. In each theme, a thematic forecast function is assigned to all cells, such that the function is a fuzzy If-Then rule to determine the infestation factor. The function for each current cell, $C_{m}$, in $\tau_{n}$, its predicted consequent infestation factor, $I_{n}$ is,

$$
\begin{aligned}
& \mathbf{f}_{\text {layer }}\left(\tau_{n}: C_{m}\right)= \\
& \text { If } \left.\left(C_{m} \text { is proximity_F } s_{n}\right) \text { Then (infestation_factor is } I_{n}\right)
\end{aligned}
$$

The thematic forecast phase has the function,

$$
\mathbf{f}_{\text {theme }}\left(T_{1}: \tau_{1}, \tau_{2}, \tau_{3}\right)=\left(T_{1}: \tau_{1,}, \tau_{2 f}, \tau_{3 f}\right)
$$

Where, $\tau_{1 f}, \tau_{2 f}$ and $\tau_{3 f}$ are the resulting themes after $\mathbf{f}_{\text {layer }}$ function has been performed on all cells at each layer of the respective themes.

\section{Phase 3: Global (Large-Scale) Forecast}

In this phase, all the layers in the previous thematic forecast, $\tau_{l f}, \tau_{2 f}, \tau_{3 f}$, is mapped onto the known infestation theme, $T_{1}$, to form a composite, $T_{2}$, to result in the forecast of infestation sites on a global scale. It must be noted that the term global does not refer to a worldwide scale, but rather to the whole given area represented on a spatial image (e.g. state, city, county). Based on parthenium weed dispersal and life-cycle parameters, inserting the themes $T_{1 f}, \tau_{1 f}, \tau_{2 f}$ and $\tau_{3 f}$ from (6) into the following global forecast function we have,

$$
\boldsymbol{f}_{\text {global }}\left(T_{1}: \tau_{l f}, \tau_{2 f}, \tau_{3, f} \tau_{\text {ull }}\right)=T_{2}
$$

Thus, for every cell in $\tau_{l f}$, $\tau_{2 f}$, and $\tau_{3 f}$, we have an implication that maps onto a corresponding cell in $T_{1}$.

$$
\tau_{l f}\left(C_{m, l f}\right) \Phi \tau_{2 f}\left(C_{m, 2 f}\right) \Phi \tau_{3 f}\left(C_{m, 3 f}\right) \rightarrow T_{l}\left(C_{m}\right)
$$

where $\Phi$ is an aggregation operator.

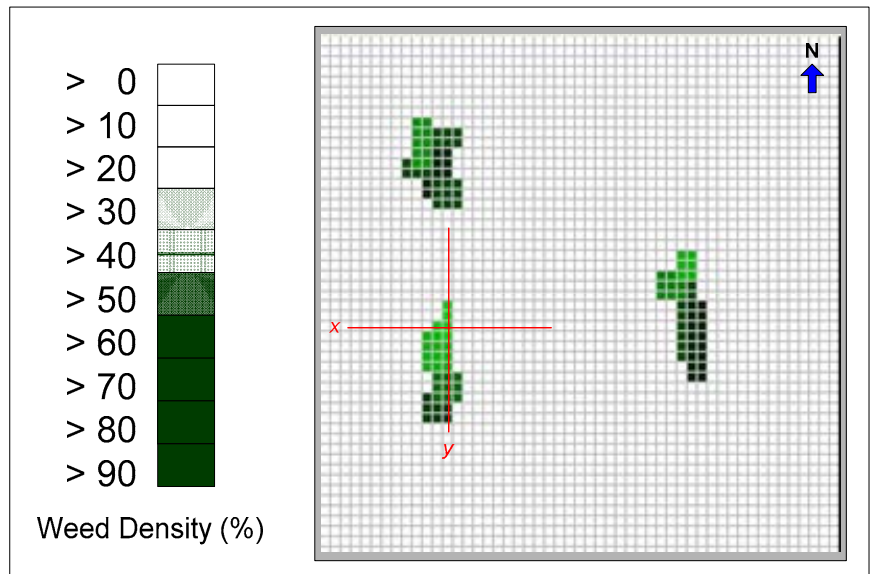

Fig. 6. : The site of known infestation at time, T1. The $\mathrm{x}$-axis and $\mathrm{y}$-axis cross section indicates samples that are taken to compare actual and predicted infestation.

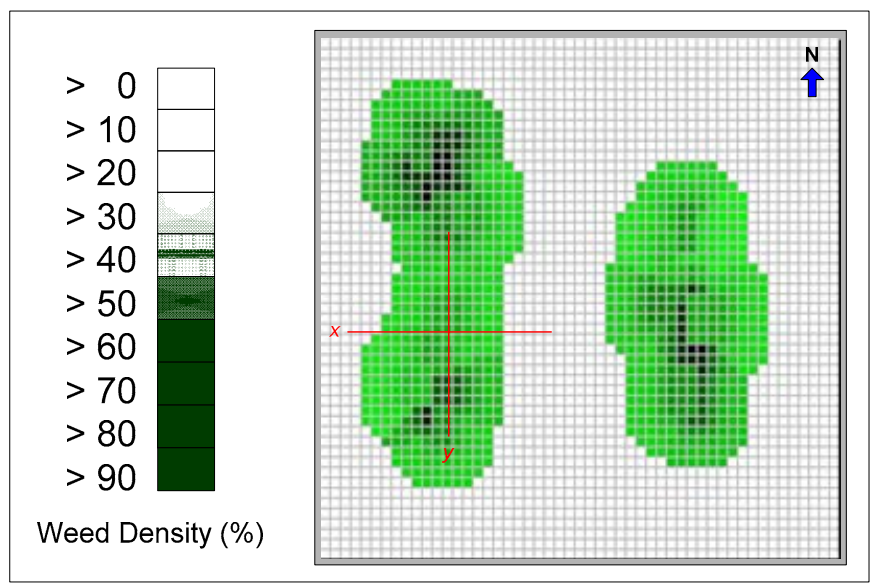

Fig. 7: Actual infestation, after approximately six months.

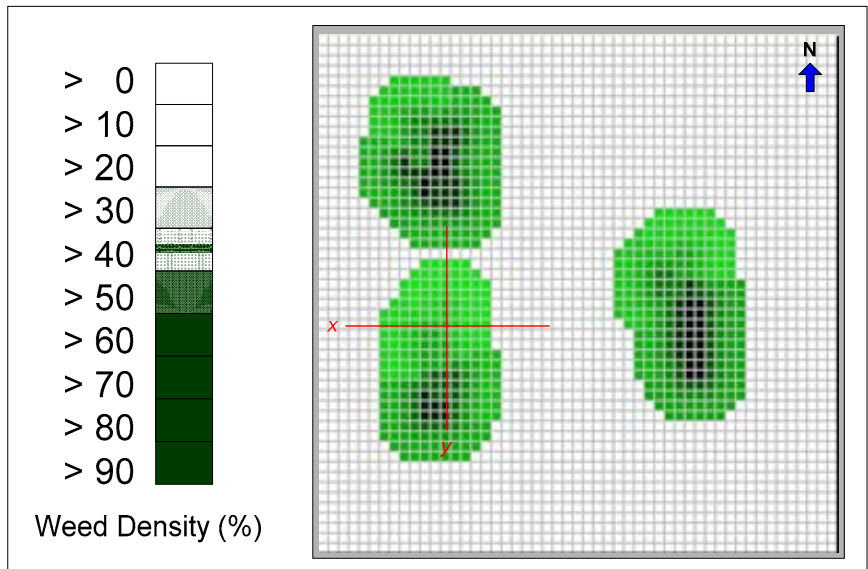

Fig. 8: Prediction dispersal, after approximately six months. 
FIGURE 9: CROSS SECTION SAMPLING OF X-AXIS OF $\mathrm{T}_{0}$, THE ORIGINAL INFESTATION, $\mathrm{T}_{\mathrm{C}}$, ACTUAL INFESTATION AND $\mathrm{T}_{6}$, PREDICTED RESULTS.

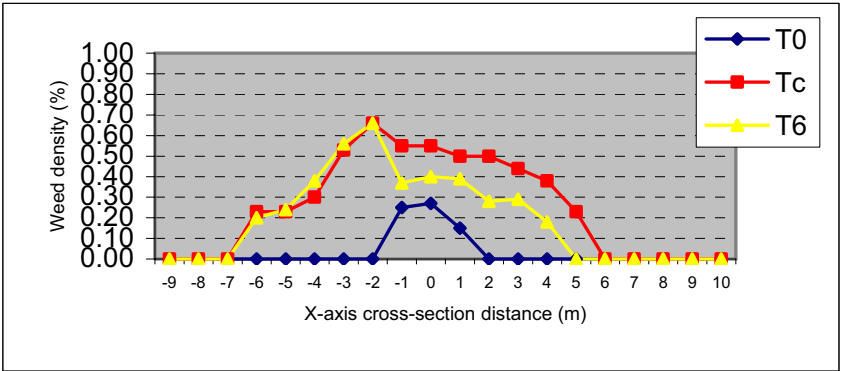

FIGURE 10: CROSS SECTION SAMPLING OF Y-AXIS OF $\mathrm{T}_{0}$, THE ORIGINAL INFESTATION, $\mathrm{T}_{\mathrm{C}}$, ACTUAL INFESTATION AND $\mathrm{T}_{6}$, PREDICTED RESULTS.

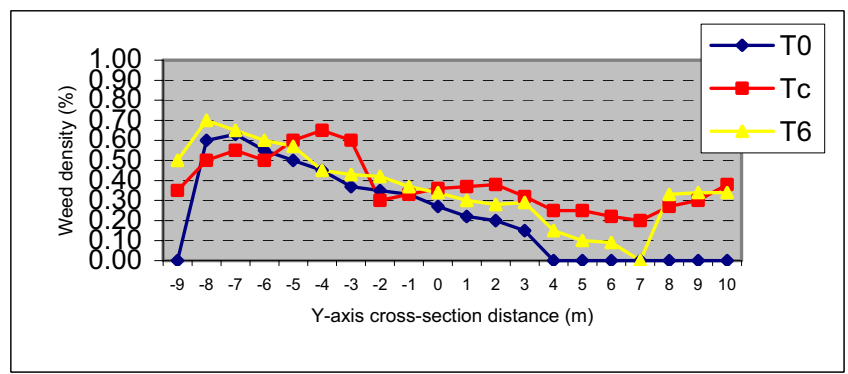

\section{Phase 4: Local Forecast}

In a global forecast, all factors influencing parthenium weed growth can mostly be categorised as static themes. Static themes normally do not and are not expected to change in the time frame of the forecasting process. However, the results from such a prediction are more useful on a global scale than for localised condition with existing local trends. As most spatial images only indicate infested sites on larger scales, it does not cover areas that are considered insignificant in the original data collection process. Coincidentally, most of these inputs coincide with factors termed as dynamic themes. These factors can be conveniently layered into individual themes as in Phase 1.

$$
\begin{aligned}
& \tau_{4}: \text { local weather } \\
& \tau_{5}: \text { local flood areas }
\end{aligned}
$$

In this case, to obtain a local forecast of parthenium infestation, these dynamic factors are mapped against the original global forecast obtained from (9). Hence, the function is,

$$
\mathbf{f}_{\text {local }}\left(\mathbf{f}_{\text {global }}\left(T_{1}: \tau_{l f}, \tau_{2 f}, \tau_{3 f,} \tau_{\text {null }}\right), \tau_{4}, \tau_{5}\right)=T_{3}
$$

\section{Test Results}

The functionality of the fuzzy post adjustment function to adapt external data acquired from remote sensors to predict dispersal of parthenium weed can be demonstrated. This is accomplished by applying its inferential process to a $50 \times 50$ meter site that has been identified as having an existing infestation (Figure 6). The actual infestation after approximately six months is shown in Figure 7. The predicted results for approximately the same duration are shown in Figure 8. Cross section results for the actual infestation and predicted results are displayed as in Figure 9 and Figure 10 respectively.

\section{SUMMARY}

This paper explored the post adjustment of input data from a remote source to fit localised weed prediction for the control and management of weed infestation. This is achieved by employing fuzzy meta-consequent functions that include a simple, multi and complex set of operators to provide the necessary capability to handle different exception types. A case study has been presented to demonstrate the workability of such fuzzy post-adjustment in the prediction of weed infestation.

\section{REFERENCES}

[1] Chippendale, J.F. and F.D. Panetta, The Cost of Parthenium Weed to the Queensland Cattle Industry, Plant Protection, 1994. 9: p. 73-76.

[2] Adamson, D.C., Determining the Economic Impact of Parthenium on the Australian Beef Industry: A Comparison of Static and Dynamic Approaches. MSC Thesis. 1996, Brisbane: University of Queensland.

[3] Chiou, A. and X. Yu, P-Expert: Integrated Expert Advisory System for Control and Management of Parthenium Weed Infestation, in Procs. 30th Annual Conference of the IEEE Industrial Electronics Society. 2004: Busan, Korea.

[4] Chiou, A. and X. Yu, P-Expert: Implementation and Deployment of Large Scale Fuzzy Expert Advisory System, in Classification and Clustering for Knowledge Discovery, S.K. Halgamuge and L. Wang, Editors. 2005, Springer-Verlag: Heidelberg.

[5] Chiou, A., X. Yu, and J. Lowry, P-Expert: A Prototype Expert Advisory System in the Management and Control of Parthenium Weed in Central Queensland, in Fuzzy Logic: A Framework for the New Millennium, V. Dimitriv and V. Korotkich, Editors. 2002, Physica-Verlag: Heidelberg.

[6] Franklin, S.E. Remote Sensing for Sustainable Forest Management. 2001, Florida, Lewis Publishers.

[7] Chamberlain, J., S. W. Dearden, A. Leitch and A. Moran. Parthenium Weed: Best Management Practice. 2000, Brisbane, DPI Publications.

[8] Chiou, A and X. Yu., Industrial Decision Support System (IDSS) in Weed Control and Management Strategies: Expert Advice Using Descriptive Schemata and Explanatory Capabilities, in Proceedings of $33^{\text {rd }}$ Annual Conference of the IEEE Industrial Electronics Society, 2007, Taipei, Taiwan.

[9] Demers, M. N. GIS Modeling in Raster. New York. 2002, John Wiley \& Sons. 